\title{
Spatial learning in preweanling rats trained in a Morris water maze
}

\author{
PHILIPP J. KRAEMER and CHRISTOPHER K. RANDALL \\ University of Kentucky, Lexington, Kentucky
}

\begin{abstract}
In three experiments, spatial learning in preweanling rats was examined, using the Morris water maze (MWM). Contrary to previous findings, Experiment 1 revealed that rat pups younger than 20 days of age showed good spatial learning. Experiment 2 revealed that spatial learning in preweanlings was influenced by the nature of the training procedure; a spaced distribution of trials resulted in good spatial learning, but a massed distribution of trials did not. Despite evidence that preweanlings have the capacity for spatial learning, Experiment 3 indicated that they are not as proficient as adults. These results are discussed in terms of procedural variables that may influence MWM performance in immature rats.
\end{abstract}

The present study was designed to test whether task variables constrain the performance of preweanling rats in the Morris water maze (MWM). Rudy, Stadler-Morris, and Albert (1987) reported a series of experiments showing that rats younger than 21 days of age were impaired on the spatial, reference memory version of the MWM. These results were interpreted as evidence of an ontogenetic constraint on spatial learning. This type of constraint is consistent with theoretical approaches to the functional development of the hippocampus (Sutherland \& Rudy, 1989), as well as with a body of related evidence on age-related limitations in information processing (Kail \& Spear, 1984; Spear \& Campbell, 1979).

There is, however, another explanation for why immature rats may not do well in the MWM. Perhaps the general procedure used by Rudy et al. (1987) and Rudy and Paylor (1988) did not provide the best conditions for demonstrating spatial learning in the young rat. In some recent experiments with adult rats, designed to assess the influence of brain damage (Prasad, Ramaiah, Baldwin, Kraemer, \& Scheff, 1994), we developed a version of the MWM task that yielded reliably good performance within a single day of training. And there are features of this procedure that led us to speculate that it might also enhance spatial learning in preweanlings.

The important features of the procedure include the apparatus and the nature of the training regime. Unlike most studies with the MWM, the apparatus consisted of a black pool placed within a dimly illuminated test room. The use of a black pool and dim illumination were intended to reduce stress and enhance processing of spatial information. In contrast to the popular trend of enriching the visibility and complexity of extramaze cues, we sought instead to exploit the relatively poor visual ca-

Please address correspondence to P. J. Kraemer, Department of Psychology, University of Kentucky, Lexington, KY 40506 (e-mail: pjkraeoo@ukcc.uky.edu). pacity of the albino rat (Munn, 1950) by constructing a set of spatial cues that were based partially on light intensity. Rather than defining spatial locations by complex distal stimuli arranged around the pool, we created a spatial intensity gradient by focusing dim spotlights differentially on the four walls of the test room. With respect to training, the procedure was designed to minimize fatigue and enhance memory processing through the use of a spaced, rather than massed, distribution of trials. Trial spacing has been shown to be a critical acquisition variable in a variety of tasks (Houston, 1991). Our goal was to provide an opportunity for the rats to rest between trials, with the delay not long enough to disrupt retention of information from one trial to the next.

\section{EXPERIMENT 1}

\section{Method}

Subjects. Thirty preweanling rats, derived from three litters, served as subjects. The litters were culled to $8-12$ pups 1 day following parturition, and the pups were group-housed with their dams in clear maternity cages. Food and water were always available in the home cages. The rats were located in a vivarium maintained on a 14:10-h light:dark cycle; all testing occurred during the light period. Training began on Postpartum Day 17, with day of birth defined as Postpartum Day 0.

Apparatus. The pool was made of a $115-\mathrm{cm}$-diam $\times 57-\mathrm{cm}-$ high circular metal tank painted flat black. The removable platform was made of a black square metal plate $(14.5 \mathrm{~cm})$, which was suspended $34 \mathrm{~cm}$ off of a metal base that rested on the floor of the pool. The pool was located in a $2.94 \times 3.87 \mathrm{~m}$ test room, $18 \mathrm{~cm}$ from the north wall, $86 \mathrm{~cm}$ from each side wall (east and west), and $233 \mathrm{~cm}$ from the other south end wall (see Figure 1).

The only light in the test room was provided by four spotlights. Two 75-W spotlights were mounted on the ceiling and projected onto the upper half of the north wall; one light was focused on the northwest corner and the other on the northeast corner. A 50-W spotlight was mounted $50 \mathrm{~cm}$ above the floor on each of the east and west walls. These lights were focused on the lower sections of the northwest and northeast corners of the room. This lighting arrangement produced a spatial gradient of light intensity around the room: more light reflected off of the north wall than off of the 


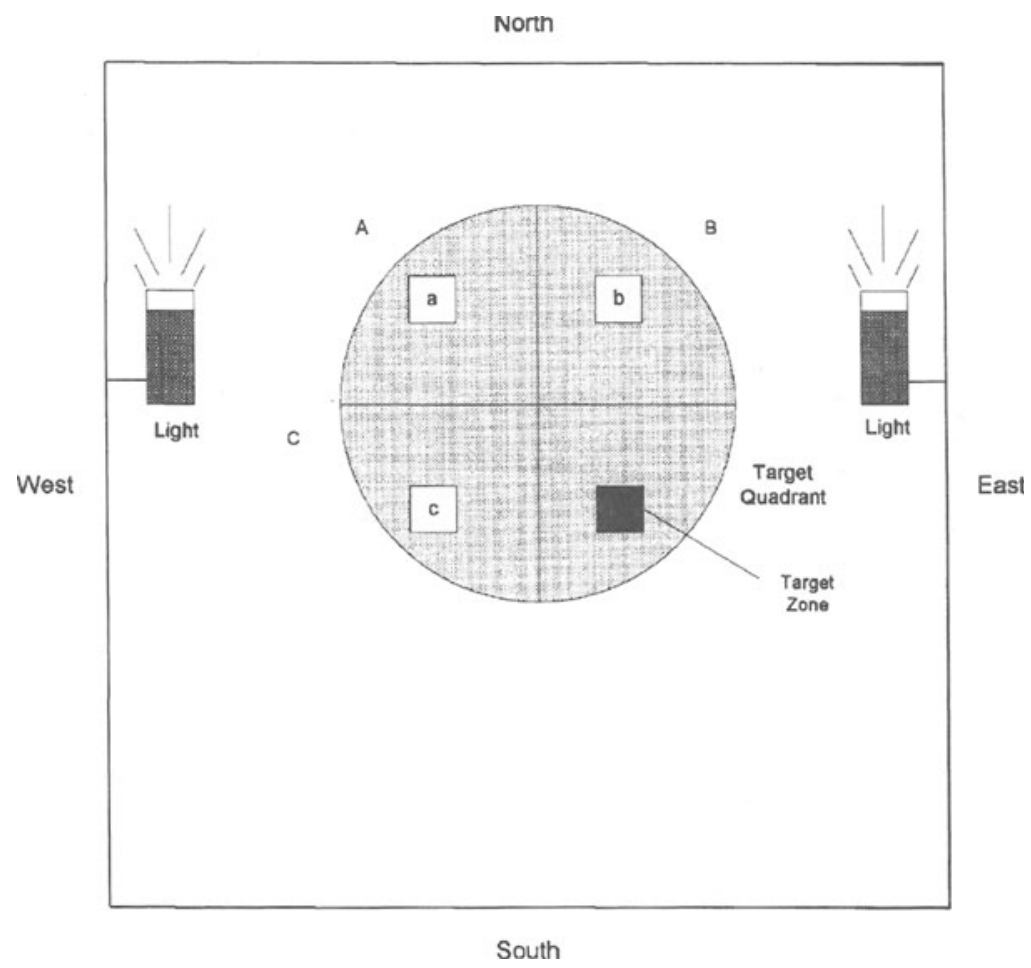

Figure 1. Schematic representation of the test pool, showing each of the four quadrants (designated A, B, C, and Target) and zones (designated a, b, c, and Target). The platform was located in the target quadrant and completely filled the target zone. Compass labels refer to points at which rats were released at the beginning of each trial.

west and east walls, which were both more illuminated than the south wall. The test-room walls also contained a variety of distal objects that served as additional spatial cues (e.g., a landscape picture on the west wall, a black pipe on the north wall, a garden hose hung on the east wall, and an assortment of laboratory furniture in front of the south wall).

The pool was divided conceptually into four quadrants, and the platform was always located in the southeast quadrant, approximately $25 \mathrm{~cm}$ from the wall of the pool (see Figure 1). The water level was maintained at $1 \mathrm{~cm}$ above the top surface of the platform, and the temperature of the water remained between $19^{\circ}$ and $21^{\circ} \mathrm{C}$. Nontoxic black powdered paint was added to the water to obscure the visual appearance of the platform.

Procedure. The subjects received two training sessions on each of 3 consecutive days. The two daily sessions were separated by a 4-h interval. Each session included four trials, separated by a 4-min intertrial interval (ITI). A trial consisted of releasing the subject from one of four compass locations surrounding the pool (north, south, east, or west) and allowing it to swim until either it climbed onto the hidden platform or $90 \mathrm{sec}$ had elapsed. The time to locate the platform was recorded (goal latency). If a subject failed to find the platform within $90 \mathrm{sec}$, it was placed there. All subjects spent $10 \mathrm{sec}$ on the platform at the end of each trial. During the ITI, the subjects were group-housed in clear maternity cages placed behind a curtain in the test room. The subjects were returned to their home cages between sessions.

Immediately after the last training trial on Day 19, the subjects received a probe trial; the hidden platform was removed, the subjects were released from the north location, and they were allowed to search for the platform for $90 \mathrm{sec}$. Each probe trial was videotaped by an overhead camera. These videotapes were analyzed by a Videomex-V motion analyzer (Columbus Instruments), which computed search times and zone visits. Search time was defined as the amount of time spent in each of the four quadrants of the pool during three consecutive 30 -sec intervals. Zone visits measured the number of times the subject entered each of four zones, equal in size to that of the escape platform. Each zone was located in a different quadrant at a relative position comparable to the placement of the platform (see Figure 1).

\section{Results}

Mean goal latencies during each of the six four-trial blocks of training are presented in Figure 2. It is clear

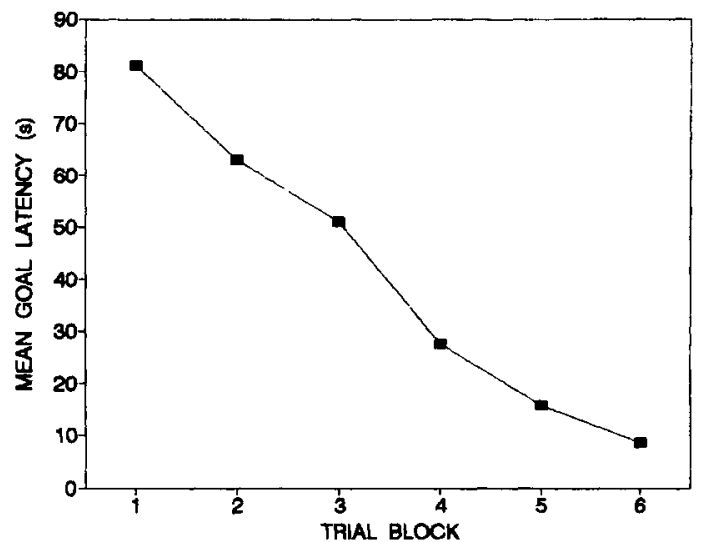

Figure 2. Mean goal latencies in Experiment 1. 


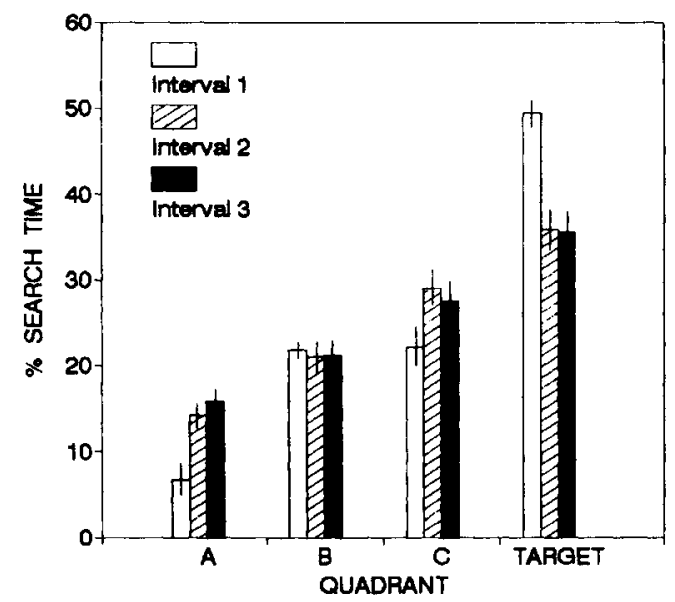

Figure 3. Mean percent search times in Experiment 1. Vertical lines indicate standard errors.

that the preweanlings exhibited a substantial improvement in finding the platform as a function of training. A repeated measures analysis of variance (ANOVA) indicated that the decrement in goal latency across trial blocks was significant $[F(4,145)=147, p<.001]$. Even stronger evidence of spatial learning was found in terms of performance during the probe trial. Mean search-time scores appear in Figure 3, plotted as percentage of time spent in each quadrant during separate $30-\mathrm{sec}$ intervals of the probe trial. The preweanlings displayed an obvious preference for the target quadrant, which had contained the platform during training, but the strength of the preference diminished over consecutive 30 -sec intervals. These observations were confirmed by a quadrant $X$ test interval ANOVA performed on percent search-time scores. There was a significant main effect of quadrant $[F(3,87)=$ 42.6, $p<.001]$ and a significant quadrant $\times$ test interval interaction $[F(6,174)=9.2, p<.001]$. Post hoc comparisons (Newman-Keuls test, $p=.05$ ) showed that more time was spent in the target quadrant during the entire $90-\mathrm{sec}$ probe trial than in any of the remaining quadrants, but the magnitude of the target quadrant preference declined over the three 30 -sec intervals.

Mean zone visits are displayed in Figure 4. The preweanlings exhibited a strong tendency to visit the target zone (where the platform had been located) more often than the other three zones (see Figure 1). The difference in visits across zones was significant $[F(3,87)=26.0$, $p<.001]$. Post hoc comparisons revealed that there were more visits to the target zone than to any of the other zones, and that there were significantly fewer visits to Zone A than to Zone B or Zone C; frequency of visits to the latter two zones were equal.

Based both on goal latency during acquisition and probe-trial performance, it is clear that the preweanlings exhibited good spatial learning. They behaved as if they had come to represent the spatial location of the hidden platform. These results contrast with those reported by Rudy et al. (1987) and Rudy and Paylor (1988). In both studies, rats younger than 20 days of age failed to show good spatial learning. It should be noted, however, that younger rats did show a decrease in goal latency over training in each study. For example, in Rudy et al. (1987, Experiment 3 ), the younger rats improved by nearly $50 \%$; despite that improvement, probe-trial performance remained well below the level indicative of good spatial learning.

There are at least two factors that may explain these conflicting results. First, there was evidence in Experiment 1 that time spent in the target quadrant changed significantly over the test period; the subjects spent more time in the target quadrant during the first $30 \mathrm{sec}$ of the probe trial than they did during the remainder of the trial. This effect is both reasonable and significant. It is not surprising to find that rats initially search an area of the pool in which they expect to find the platform and then move away from that area when it is not found. The interpretational importance of this tendency is that it is possible to underestimate what the rat knows about the location of the platform. Depending on a rat's criterion for abandoning a quadrant, a preference for the platform location may be obscured by how the rat reacts to a failed search.

Another important factor involves procedural differences among the studies. One set of procedural variables involves the visual properties of the test situation. The conventional MWM procedure relies on a white pool filled with white cloudy water located in a well-illuminated test room. Spatial cues typically consist of a rich array of distal objects arranged around the pool. The spatial environment often includes furniture, laboratory paraphernalia, and complex patterns and forms attached to the walls of the test room. Our strategy, in contrast, was to test with a black pool, filled with black cloudy water, located in a dimly illuminated test room. We attempted to enhance the functional quality of extramaze spatial cues by utilizing dim spotlights that not only illuminated different distal stimuli on the test room walls, but also provided a spatial gradient of illumination. We speculate that

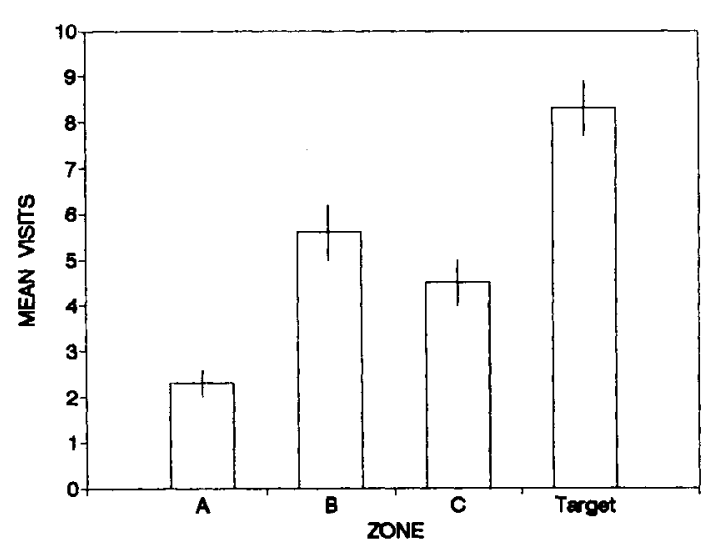

Figure 4. Mean zone visits in Experiment 1. Vertical lines indicate standard errors. 
these test conditions might diminish stress and facilitate processing of spatial information, including both visual and intensity cues.

The second set of variables includes those aspects of the procedure that affect memory processing and general performance. Such factors as trial duration, intertrial interval, and the organization and distribution of trials could all influence learning. Perhaps some conditions are more conducive to spatial learning than others. Especially important is the need to balance a concern for the physical demands imposed by the task with conditions that affect memory processing. In Experiment 1, for example, we used a distribution of trials that was designed to allow the preweanlings time to recover from the fatigue and stress associated with swimming in a novel environment, but yet did not space the trials so far apart as to enhance forgetting across trials.

\section{EXPERIMENT 2}

Experiment 2 examined directly the possibility that procedural variables influence MWM performance in preweanlings. We attempted to compare the general procedure used in Experiment 1 with a procedure that included many, but not all, of the salient aspects of the procedure used by Rudy et al. (1987). For descriptive purposes, we refer to the procedure used in Experiment 1 as spaced and that used by Rudy et al. as massed, given that the most important differences between the two involved the distribution of trials. All rats received two training sessions, presented on consecutive days. The massed procedure consisted of 12 trials presented each session, separated by a short (35- to 45-sec) ITI. Each session with the spaced procedure consisted of two four-trial blocks, separated by $4 \mathrm{~h}$, with a 5 -min interval between trials within a block.

If spatial learning in preweanlings is evident with both procedures, then we can infer that there is something about the apparatus and lighting conditions that seems to enhance performance in immature rats; if, however, we observe evidence of spatial learning only with the spaced procedure, then we can conclude that task variables are important determinants of MWM performance in preweanlings.

\section{Method}

Subjects and Apparatus. Twenty preweanling rats derived from two litters served as subjects in this experiment, and they were housed exactly as described in Experiment 1 . The subjects were randomly divided into two groups, with each litter equally represented in each group. Both groups began training on Postpartum Day 18. The apparatus was the same as that used in Experiment 1.

Procedure. Subjects in Group S (spaced training procedure) were trained with the same procedure used in Experiment 1, with the exception that training continued for 2 rather than 3 days. Subjects in Group $\mathrm{M}$ (massed training procedure) were trained in a manner similar, but not identical, to that described by Rudy et al. (1987, Experiment 3). Unlike Group S, they were given pretraining at the beginning of the first acquisition session. Pretraining began by placing the subject onto the platform for $30 \mathrm{sec}$. It was then removed and trained to climb onto the platform, once from each side, by placing only its front paws on the platform. Finally, an initial 30-sec swimming trial was completed, in which the subject was guided to the platform. Training began immediately after completion of pretraining. Each training trial was separated by a 35- to 45-sec ITI, which was spent on the platform. The pups were released into the pool once from each of the four compass points within each consecutive sequence of four trials, with the order of release randomized within each sequence for each pup. The maximum trial duration for subjects in Group $M$ was 60 sec. Probe trials, presented $15 \mathrm{~min}$ after the last training trial, were the same for both groups and were conducted as described in Experiment 1. Goal latencies were measured during acquisition and both search times and zone visits were measured during the probe trial.

\section{Results}

Goal latency. Mean goal latencies for each four-trial block of training are presented in Figure 5. Given the vast differences in the training regimes, goal latencies were analyzed separately for each group. Group S showed a precipitous decline in goal latency over training. A repeated measures ANOVA indicated that this decrement was significant $[F(3,27)=31.3, p<.001]$. Post hoc comparisons showed that mean goal latency during each trial block differed significantly from that of every other
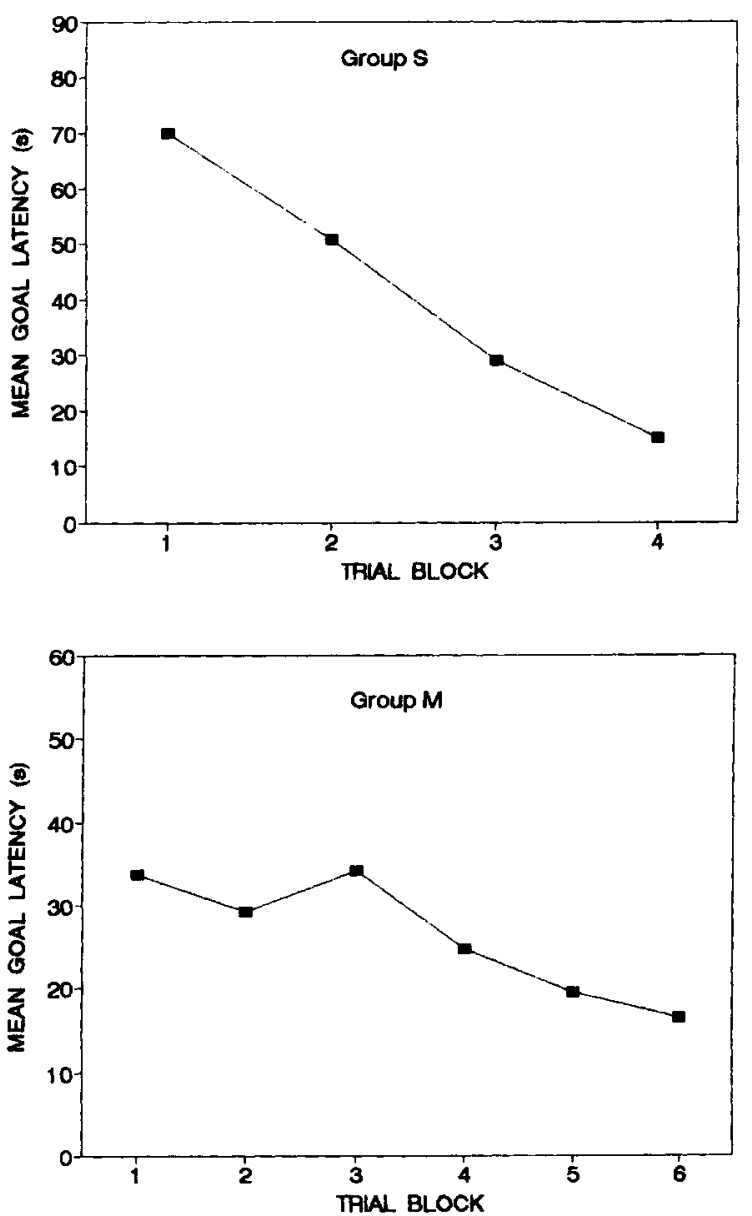

Figure 5. Mean goal latencies for Groups $M$ (massed trials) and $S$ (spaced trials) in Experiment 2. 
trial block. Although Group $M$ was trained with 12 trials massed together, we again examined acquisition in terms of blocks of four trials, comparable to the analysis for Group S. This group also exhibited a decline in goal latency over trial blocks, but the decrement appeared to be more irregular and less dramatic than that found with Group S. The decline in goal latency over trial blocks was, however, significant $[F(5,45)=7.4, p<.001]$. Post hoc tests indicated that goal latencies on Trial Blocks 5 and 6 were equal and significantly lower than those on Trial Blocks 1 and 2, which were equal. No other difference was significant among the trial blocks for Group M.

Probe-trial performance. Mean percent search times and zone visits are summarized for both groups in Figures 6 and 7, respectively. With respect to search times, Group $\mathrm{S}$ displayed a preference for the target quadrant that diminished in magnitude over consecutive $30-\mathrm{sec}$ intervals. A quadrant $\times$ test interval (three 30 -sec intervals) ANOVA performed on the percent search-time scores indicated a significant main effect for quadrant
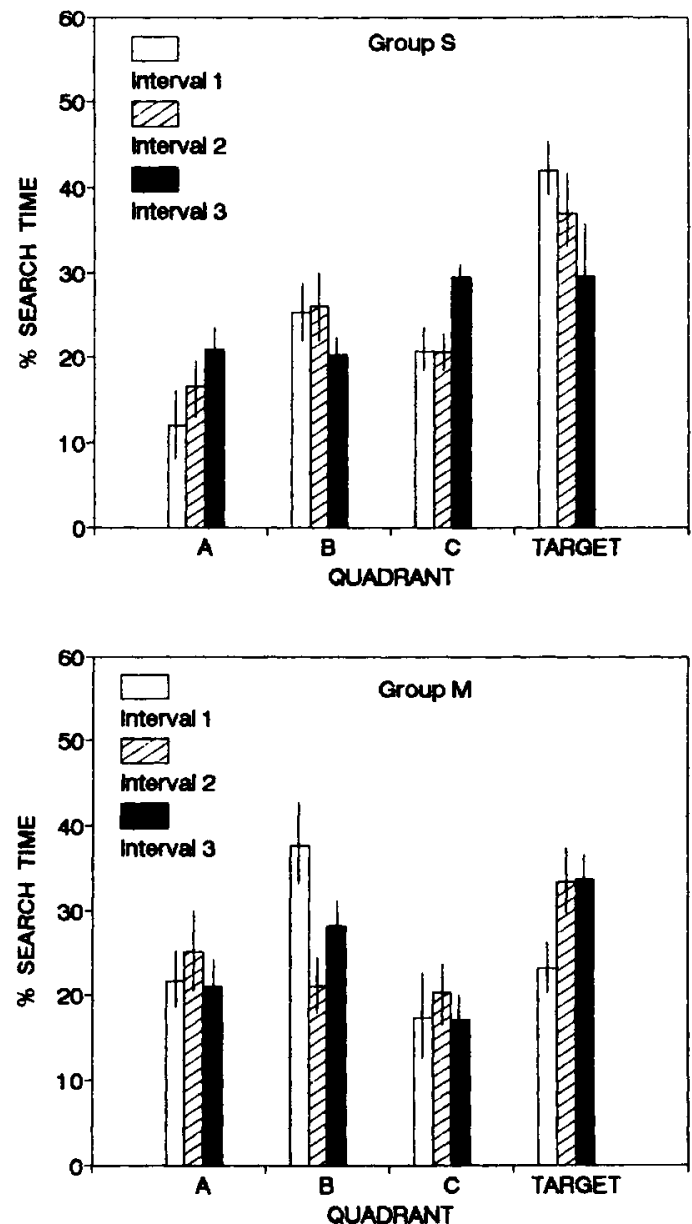

Figure 6. Mean search times for Groups M (massed trials) and S (spaced trials) in Experiment 2. Vertical lines indicate standard errors.
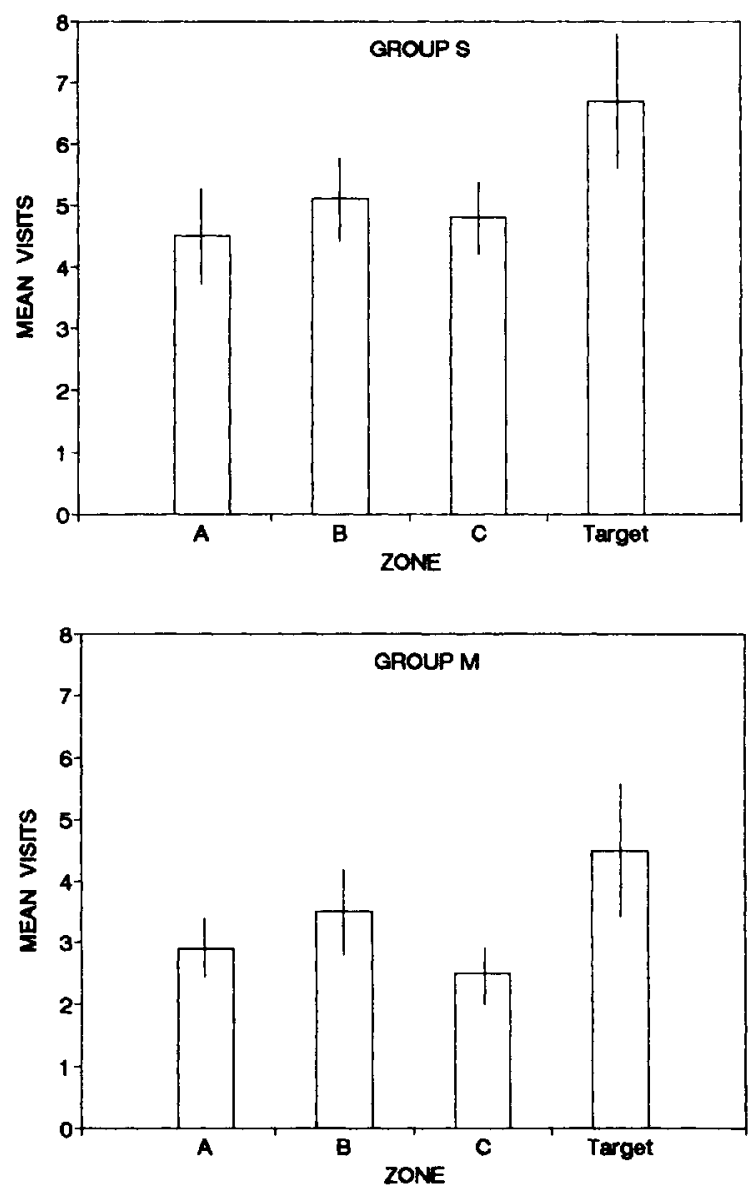

Figure 7. Mean zone visits for Groups $M$ (massed trials) and S (spaced trials) in Experiment 2. Vertical lines indicate standard errors.

$[F(3,27)=5.1, p<.01]$ and a significant quadrant $\times$ interval interaction $[F(6,54)=3.6, p<.01]$. Simple main effects analyses showed that the interaction was the result of a change in the time spent in the A, C, and target quadrants (see Figure 1) over the three test intervals; time spent in the target quadrant declined $[F(2,18)=3.7, p<$ $.05]$, whereas time spent in the $\mathrm{A}$ and $\mathrm{C}$ quadrants increased $[F(2,18)=3.7, p<.05$, and $F(2,18)=9.2, p<$ .01 , respectively]. A different pattern of results emerged for Group M. These preweanlings displayed only a weak nonsignificant preference for the target quadrant. The quadrant $\times$ test interval ANOVA performed on the percent search-time scores failed to yield a significant main effect or interaction.

In order to directly compare performance of the two groups, we also analyzed percent search-time scores for the target quadrant across the three $30-$ sec test intervals. This analysis yielded a significant group $X$ interval interaction $[F(2,36)=9.7, p<.001]$. Post hoc comparisons indicated that percent search times were significantly higher in Group S during the first 30-sec interval but not during the remaining two intervals. Finally, neither group showed a significant difference in the number 
of visits to the four zones, nor was there a significant difference between the two groups in the number of visits to the target zone.

\section{Discussion}

This pattern of results indicates that spatial learning was enhanced in Group S relative to Group M. Preweanlings trained with the spaced procedure displayed a dramatic decline in latency to locate the hidden platform during training, and their behavior during the probe trial supports the inference that they had actually acquired knowledge of the spatial position of the platform. Although preweanlings trained with the massed procedure showed improvement in finding the platform over training, they did not express a significant preference for the target quadrant during the probe trial.

The absence of a significant difference between the two groups on the zone-visit measure, in combination with lower search-time scores for Group $S$ than those obtained in Experiment 1, implies that the magnitude of spatial learning was less pronounced than that obtained in the first experiment. The fewer number of trial blocks in the second experiment is a reasonable explanation for that difference.

Our general conclusion is that Group M behaved similarly to the preweanlings tested in Rudy et al. (1987) and Group $S$ behaved similarly to the preweanlings tested in our first experiment. Given that the same apparatus and test environment were used for both groups, it appears that the nature of the training procedure influenced spatial learning, although it remains to be shown whether or not the spaced procedure would be equally effective with a more traditional apparatus and test environment.

There is extensive evidence that acquisition in many learning tasks is influenced by the duration, number, and distribution of training trials (Fanselow \& Tighe, 1988; Houston, 1991). Unfortunately, there is little evidence available concerning the influence of task variables on MWM performance. Our hypothesis is that proficiency on the MWM requires that the training procedure balance variables that affect information processing directly with those that affect it indirectly. For example, trial spacing will affect retention of information from one trial to the next; if trials are spread too far apart, the subject may forget and be unable to construct an effective spatial representation. Conversely, if insufficient time is allowed between trials for the subject to recover from the stress and fatigue of swimming, then memory processing may be disrupted. This concern is especially relevant for the preweanlings, who may be more physically challenged than adults by task demands associated with the MWM.

With our spaced procedure, we believe that we have struck a good balance. The subjects are allowed sufficient time to recover between trials without the delay's being so long as to induce forgetting.

Another important difference between the procedures used with Groups $S$ and $M$ involves trial duration. Group S was trained with trials that were, at a maximum, $90 \mathrm{sec}$ in duration; maximum trial duration for Group $\mathrm{M}$, as in the Rudy et al. (1987) study, was set at $60 \mathrm{sec}$. This difference could have an effect on spatial learning by increasing the reward value of finding the platform. On the first trial block, subjects in Group $S$ had a potentially longer time to search for the platform than did those in Group M. For Group S, greater fatigue, resulting from more swimming, could have enhanced the reward value of escaping onto the platform, or it could have allowed the subject more exposure to the visual environment, thereby enhancing processing of spatial cues. Obviously, additional research is required to characterize the degree to which procedural variables impact MWM performance in preweanlings, as well as in adults.

\section{EXPERIMENT 3}

The first two experiments established that preweanlings younger than 20 days of age were capable of exhibiting good spatial learning in a MWM. It remained to be shown whether the level of performance expressed by preweanlings differed from that of adults. This experiment directly compared the performance of preweanlings and adults trained with either a spaced or a massed procedure. Unlike the previous experiments, all training occurred on a single day. Although adults show very good spatial learning when trained in this way (Prasad et al., 1994), it was questionable whether three blocks of trials, all presented on the same day, would be sufficient to produce learning in preweanlings.

\section{Method}

Subjects and Apparatus. Twenty-two preweanlings, procured from two litters, and 20 adult rats were used in this study. The apparatus was the same as that used in Experiments 1 and 2.

Procedure. All MWM training occurred on Postpartum Day 18. Spaced-training subjects (Group $\mathrm{S}$ ) received three sessions of four trials each. The sessions were separated by $2 \mathrm{~h}$, and trials within a session were separated by 4 min. Massed-training subjects (Group $\mathrm{M}$ ) received 12 consecutive acquisition trials separated by a 4-min ITI. All subjects spent the ITI with littermates in clear maternity cages located behind a curtain in the test room. Subjects in both groups were released once from each of the four compass points within each consecutive sequence of four trials. A probe test was conducted immediately after the last training trial.

\section{Results}

Mean goal latencies are plotted separately for preweanlings and adults in Figure 8, with scores for spaced and massed groups combined. Both age groups showed a decrement in goal latency over training, but preweanlings were clearly slower than adults to find the hidden platform. Because age is confounded with size, which affects swimming speed, we analyzed goal latencies separately at the two ages. Adults showed a significant decrease in goal latency over trial blocks $[F(2,36)=62.1$, $p<.001]$. Post hoc comparisons revealed a significant decline in goal latency between Trial Blocks 1 and 2 and between Trial Blocks 2 and 3. Preweanlings also displayed a significant decrease in goal latency over trial 


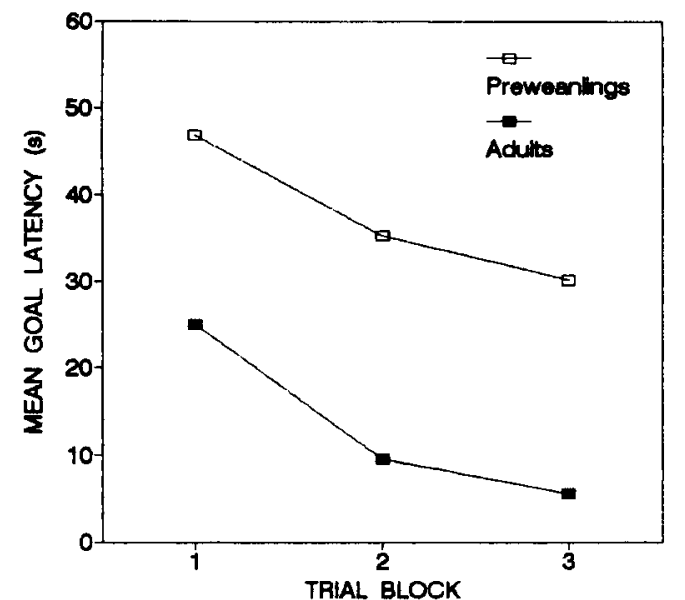

Figure 8. Mean goal latencies for preweanlings and adults in Experiment 3.

blocks $[F(2,40)=15.1, p<.001]$. Unlike adults, however, preweanlings did not improve significantly between Trial Blocks 2 and 3, although the decline in goal latency between Trial Blocks 1 and 2 was significant. There was no significant difference in goal latency between groups given spaced and massed training at either age.

Mean percent search-time scores for preweanlings and adults appear in Figure 9. Preweanlings displayed a weak preference for the target quadrant, especially during the first two intervals, whereas adults expressed a strong preference for that quadrant. These observations were confirmed by separate group (spaced or massed training) $\times$ quadrant $\times$ test interval ANOVA performed on percent search-time scores at each age. For preweanlings, there was no significant main effect or interaction; time spent in the target quadrant did not differ from time spent in other quadrants at any of the three test intervals for either the spaced or massed group. In adults, there was a significant main effect of quadrant $[F(3,54)=32.3$, $p<.001]$, and the quadrant $\times$ test interval interaction was also significant $[F(6,108)=3.0, p<.05]$. There was no significant main effect of group, nor were any of the other interactions significant. Post hoc tests revealed that the main effect of quadrant was the result of adults having spent significantly more time in the target quadrant than in other quadrants over all three test intervals. The significant quadrant $\times$ test interval interaction was primarily due to slight changes in the percent of time spent in quadrants $\mathrm{A}$ and $\mathrm{C}$ during Test Interval 2; time in $\mathrm{A}$ increased and time in $\mathrm{C}$ decreased during the second test interval. Time in the target quadrant did not change significantly over the three test intervals.

Mean zone visits, displayed in Figure 10, were analyzed by an age $\times$ group (spaced or massed) $\times$ zone (each of the four zones displayed in Figure 1) ANOVA. The age $\times$ zone interaction was significant $[F(3,57)=15.1, p<$ $.001]$. Among preweanlings, there was a significant difference in visits to the four zones $[F(3,63)=6.8, p<$ $.001]$. Post hoc comparisons indicated that there were equal visits to the target zone and the $\mathrm{C}$ zone, and more visits to these two zones than to Zones $A$ and $B$, which accrued equal visits. None of the other pairwise comparisons was significant. Adults also showed a significant difference in visits to the various zones $[F(3,57)=$ $38.6, p<.001]$, but the pattern of performance was somewhat different from that of the preweanlings. Adults visited the target zone more often than any other zone, and they visited Zone $\mathrm{C}$ more often than Zones $\mathrm{A}$ and $\mathrm{B}$; visits to the latter two zones were equal. Finally, performance across the two age groups was compared by analyzing visits to the target zone alone. Significantly more target zone visits occurred in adults than in preweanlings $[F(1,38)=43.6, p<.001]$.

\section{Discussion}

Although adult rats performed quite well when trained within 1 day, preweanlings were comparatively deficient. With respect to acquisition, a decrement in goal latency was evident across the three trial blocks at each age. In adults, the decline was steady and pronounced, whereas in preweanlings, it was regular, but much less robust. There were also absolute differences in goal la-
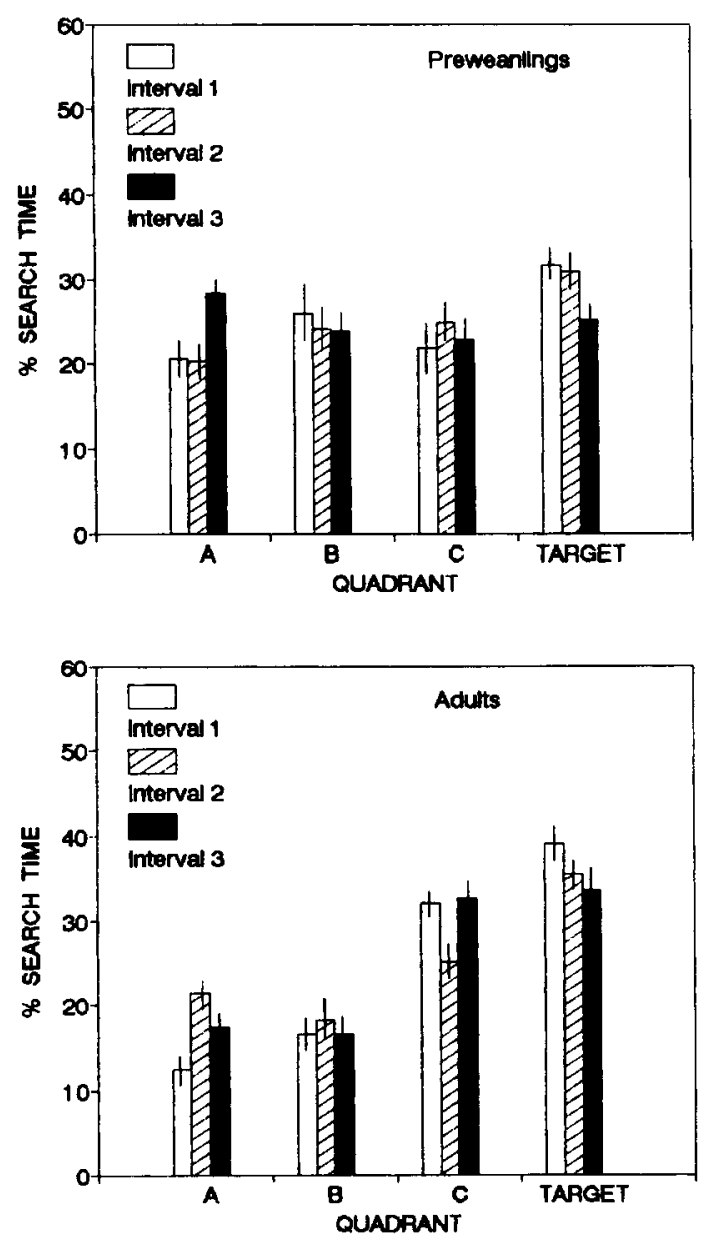

Figure 9. Mean search times for preweanlings and adults in Experiment 3. Vertical lines indicate standard errors. 

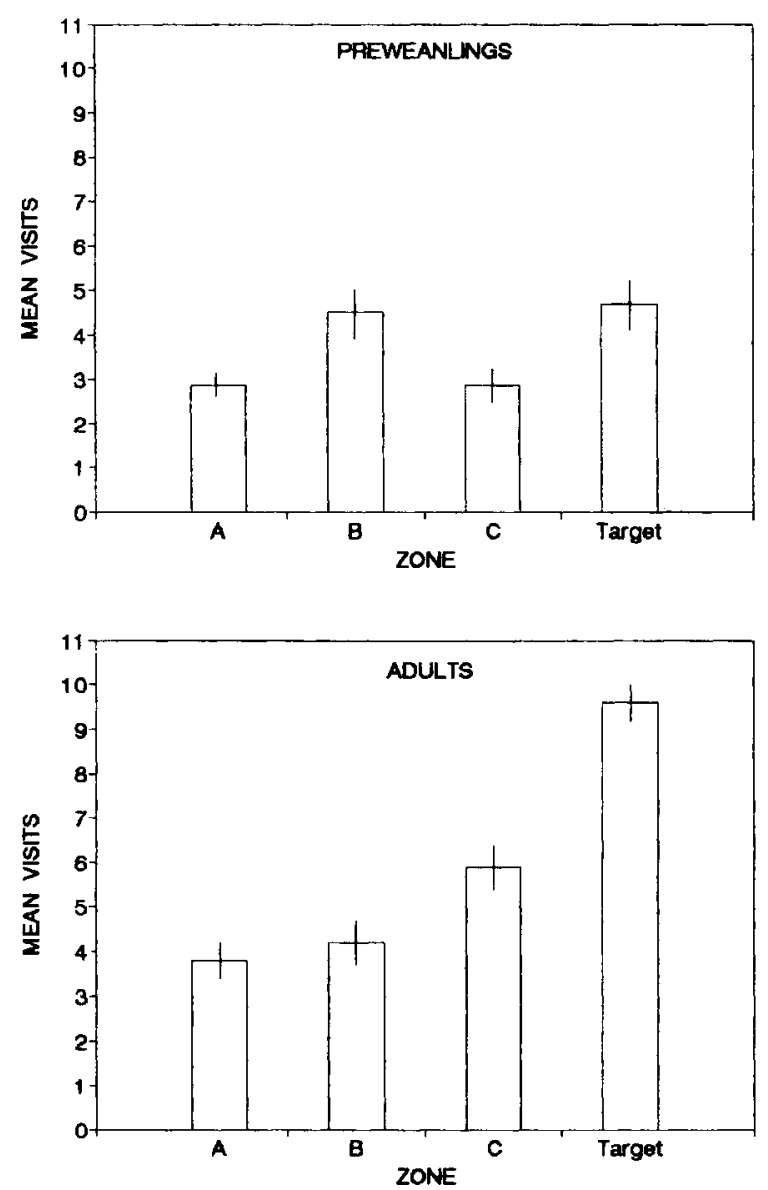

Figure 10. Mean zone visits for preweanlings and adults in Experiment 3. Vertical lines indicate standard errors.

tency between the two ages. At each trial block, adults reached the platform much more quickly than did preweanlings - an effect that can be attributed to the impact of size and motor facility. What is more interesting, however, is that preweanlings never achieved a level of performance as good as that found in Experiments 1 and 2. Mean goal latency during Trial Block 3 remained high, relative to the levels achieved by preweanlings in the other two experiments. This difference could indicate that good performance on the MWM in preweanlings requires more than a single day of training, more than 12 trials of training, a longer interval between sessions, or some combination of these factors.

Another difference is that preweanlings in this experiment did not show a strong preference for the target quadrant or target zone with either a spaced or a massed distribution of trials. In contrast, adults displayed a strong target quadrant and target zone preference that was equal for groups trained with massed and spaced procedures. Thus, although a single day of training produces good spatial learning in adults, it appears that it is not sufficient for preweanlings. There was also no indication at either age that performance with the single-day procedure was affected by the spacing and distribution of trials.

\section{GENERAL DISCUSSION}

The important conclusion of this study is that rats younger than 20 days of age are capable of spatial learning in the MWM. In Experiments 1 and 2, rats tested at 19 days of age showed good spatial learning, as indicated by both a decline in goal latency during training and a significant preference for the target quadrant and the target zone expressed during the probe trial. Despite the ability to show spatial learning, rats at this age were still not as proficient as adults.

In contrast to our findings, both Rudy et al. (1987) and Rudy and Paylor (1988) found that rats did not perform well on the MWM until they were older than 20 days of age. One obvious possibility is that the discrepant results reflect strain differences. Our experiments tested Sprague-Dawley-derived rats, whereas Rudy and his colleagues tested Long-Evans hooded rats. Unfortunately, there has been too little attention in general given to ontogenetic comparisons between strains. Thus, we do not know whether neural systems that subserve spatial learning functionally mature at different rates in the two strains. Our results imply that, at least for SpragueDawleys, these neural systems are functioning before 20 days of age, although perhaps less proficiently than in adults.

This conclusion is contingent on the assumption that preweanlings in this study did, indeed, exhibit spatial learning. It has been suggested that preweanlings might have learned instead to locate the hidden platform by relying on a landmark strategy (McNaughton, Chen, \& Markus, 1991; O’Keefe \& Nadel, 1978). For example, perhaps preweanlings learned to approach a specific level of illumination, instead of representing a spatial configuration. ${ }^{1}$ Although it is difficult to rule out the use of a nonspatial learning strategy in the MWM, we believe that our methodology and results argue against such a possibility. Consistent with conventional methods, we sought to create a spatial task by releasing subjects from different locations during each trial block. This procedure prevents the rat from using a simple motor sequence to find the platform. It also changes the perception of distal cues, which reduces the opportunity to solve the task by approaching discrete stimuli associated with specific spatial locations. The results relevant to this issue are the two probe-trial measures we reported, which are typically used to infer spatial learning in the MWM. The logic is that if a rat spends more time in the quadrant that had contained the platform, and if it swims through the precise area previously occupied by the platform more often than it swims through other areas of the pool, then it can be inferred that performance was guided by a spatial representation. This was the case in Experiments 1 and 2.

Our conclusion therefore is that preweanlings did exhibit good spatial learning in Experiments 1 and 2. We 
also accept as valid and reliable failures to discover spatial learning at this age (Rudy \& Paylor, 1988; Rudy et al., 1987) - a pattern of results that we replicated in Experiment 3. We speculate further that our positive results are due to methodological factors rather than to any inconsistency in the phenomenon itself.

There are three salient features of our procedure that are worth considering. First, we used a dimly illuminated room, in which there was a spatial gradient of light intensity provided by variations in light reflected from the four walls surrounding the pool. Rather than attempting to highlight extramaze distal objects, we provided a visual environment that might make it easier for the rat to identify spatial locations.

Second, our initial procedure used a longer trial duration, greater intertrial interval, and a more dispersed distribution of trials than has typically been used with the MWM. This combination of training parameters may both promote memory storage and limit the adverse impact of peripheral performance factors, such as fatigue and stress. This aspect of our procedure was partially evaluated in Experiment 2. Two groups of preweanlings were trained with the same apparatus: one group followed the general procedure used in Experiment 1 and the other group followed the general procedure used by Rudy et al. (1987). Despite having received fewer trials overall, only the former group (spaced distribution of trials) behaved during the probe trial as if they had learned the location of the platform. Due to other procedural differences between the two groups, however, we cannot conclude that the spatial learning exhibited by preweanlings was a consequence of trial spacing alone. Nor can we conclude that the apparatus and visual test conditions alone guarantee good spatial learning in preweanlings. If that were the case, then both spaced and massed groups in Experiment 2 would have performed well.

Finally, our measurement of probe-trial performance included a simple modification that might be important. Rather than analyzing quadrant preference over the entire probe trial, we divided the test period into three 30 $\mathrm{sec}$ intervals. The rationale was that rats may stay in the target quadrant initially but that, as they fail to find the platform, they may begin to search in other locations. This behavioral tendency could obscure an overall preference for the target quadrant.

There is one final issue that deserves discussion. In Experiments 2 and 3, and also in Rudy et al. (1987), there was evidence of a performance dissociation: preweanlings showed a substantial decrement in goal latency during training, but still failed to express a preference for the target quadrant during the probe trial. One interpretation of this dissociation is that it simply reflects the differential sensitivity of the two measures to degree of learning; more training may be required in order to de- tect learning with the search-time measure than with the goal-latency measure. Another interpretation is that learning with the MWM involves several components which depend on different processes. A decline in goal latency during acquisition might not depend entirely on the establishment of a spatial representation. Goal latencies might decline for several reasons, including the abandonment of inefficient search strategies such as circling around the perimeter. Whatever the source of this performance dissociation, it deserves additional attention. It might prove to be especially valuable for diagnostic analysis of brain injury that rely on the MWM as an index of cognitive function (Brandeis, Brandys, \& Yehuda, 1989; Smith, Okiyama, Thomas, Claussen, \& McIntosh, 1991).

\section{REFERENCES}

Brandeis, R., Brandys, Y., \& Yehuda, S. (1989). The use of the Morris water maze in the study of memory and learning. International Journal of Neuroscience, 48, 29-69.

Fanselow, M. S., \& Tighe, T. J. (1988). Contextual conditioning with massed versus distributed unconditional stimuli. Journal of Experimental Psychology: Animal Behavior Processes, 14, 187-199.

Houston, J. P. (1991). Fundamentals of learning and memory. New York: Harcourt Brace Jovanovich.

KAIL, R., \& SPEAR, N. E. (1984). Comparative perspectives on the development of memory. Hillsdale, $\mathrm{NJ}$ : Erlbaum.

McNaughton, B. L., Chen, L. L., \& Markus, E. J. (1991). "Dead reckoning," landmark learning, and the sense of direction: A neuropsychological and computational hypothesis. Journal of Cognitive Neuroscience, 3, 190-202.

MUNN, N. L. (1950). Handbook of psychological research on rat. Boston: Houghton Mifflin

O'KeEFE, J., \& NADEL, L. (1978). The hippocampus as a cognitive map. Oxford: Oxford University Press, Clarendon Press.

Prasad, M. R., Ramaiah, C., Baldwin, S. A., Kraemer, P. J., \& SCHEFF, S W. (1994). Characterization of lateral cortical impact brain injury in the rat. Manuscript submitted for publication.

RUDY, J. W., \& PAYLOR, R. (1988). Reducing the temporal demands of the Morris place-learning task fails to ameliorate the place-learning impairment of preweanling rats. Psychobiology, 16, 152-156.

RudY, J. W., STADler-MorRIS, S., \& AlberT, P. (1987). Ontogeny of spatial navigation behaviors in the rat: Dissociation of "proximal"and "distal"-cue-based behaviors. Behavioral Neuroscience, 101, $62-73$

Smith, D. H., Okiyama, K., Thomas, M., Claussen, B., \& McInTosH, T. (1991). Evaluation of memory dysfunction following experimental brain injury using the Morris water maze. Journal of Neurotrauma, 8, 259-269.

SPEAR, N. E., \& Campbell, B. A. (1979). Ontogeny of learning and memory. Hillsdale, NJ: Erlbaum.

Sutherland, R. J., \& RUDY, J. W. (1989), Configural association theory: The role of the hippocampal formation in learning, memory and amnesia. Psychobiology, 17, 129-144.

\section{NOTE}

1. This possibility was suggested by an anonymous reviewer.

(Manuscript received November 12, 1993; revision accepted for publication October 20, 1994.) 